\title{
Tolerance Factor and Cooperative Tilting Effects in Vacancy-Ordered Double Perovskite Halides
}

\author{
Annalise E. Maughan, ${ }^{\dagger}$ Alex M. Ganose, ${ }^{\ddagger, \Phi, \S}$ Mohammed A. Almaker, ${ }^{\dagger}$ David O. Scanlon, ${ }^{\ddagger}$, I, $\S_{(0)}$ \\ and James R. Neilson* $*+\infty$ \\ ${ }^{\dagger}$ Department of Chemistry, Colorado State University, Fort Collins, Colorado 80523-1872, United States \\ ${ }^{\ddagger}$ Kathleen Lonsdale Materials Chemistry, Department of Chemistry, University College London, 20 Gordon Street, London WC1H \\ 0AJ, United Kingdom \\ II Diamond Light Source Ltd., Diamond House, Harwell Science and Innovation Campus, Didcot, Oxfordshire OX11 0DE, United \\ Kingdom \\ ${ }^{\S}$ Thomas Young Centre, University College London, Gower Street, London WC1E 6BT, United Kingdom
}

\section{Supporting Information}

\begin{abstract}
Lattice dynamics and structural instabilities are strongly implicated in dictating the electronic properties of perovskite halide semiconductors. We present a study of the vacancy-ordered double perovskite $\mathrm{Rb}_{2} \mathrm{SnI}_{6}$ and correlate dynamic and cooperative octahedral tilting with changes in electronic behavior compared to those of $\mathrm{Cs}_{2} \mathrm{SnI}_{6}$. Though both compounds exhibit native $n$-type semiconductivity, $\mathrm{Rb}_{2} \mathrm{SnI}_{6}$ exhibits carrier mobilities that are reduced by a factor of $\sim 50$ relative to $\mathrm{Cs}_{2} \mathrm{SnI}_{6}$. From synchrotron powder X-ray diffraction, we find that $\mathrm{Rb}_{2} \mathrm{SnI}_{6}$ adopts the tetragonal vacancy-

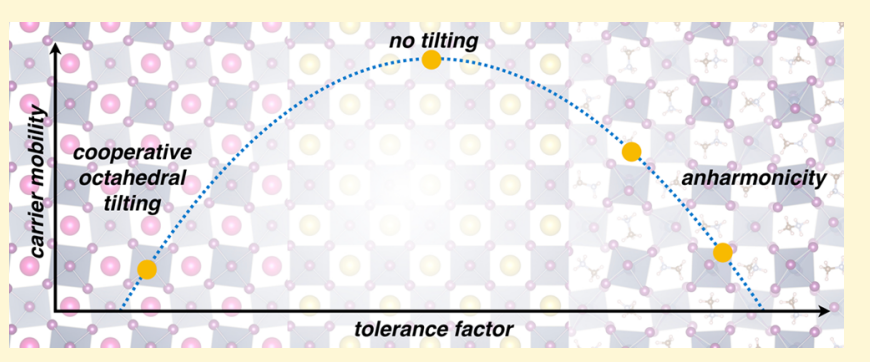
ordered double perovskite structure at room temperature and undergoes a phase transition to a lower-symmetry monoclinic structure upon cooling, characterized by cooperative octahedral tilting of the $\left[\mathrm{SnI}_{6}\right]$ octahedra. X-ray and neutron pair distribution function analyses reveal that the local coordination environment of $\mathrm{Rb}_{2} \mathrm{SnI}_{6}$ is consistent with the monoclinic structure at all temperatures; we attribute this observation to dynamic octahedral rotations that become frozen in to yield the low-temperature monoclinic structure. In contrast, $\mathrm{Cs}_{2} \mathrm{SnI}_{6}$ adopts the cubic vacancy-ordered double perovskite structure at all temperatures. Density functional calculations show that static octahedral tilting in $\mathrm{Rb}_{2} \mathrm{SnI}_{6}$ results in marginally increased carrier effective masses, which alone are insufficient to account for the experimental electronic behavior. Rather, the larger number of low-frequency phonons introduced by the lower symmetry of the $\mathrm{Rb}_{2} \mathrm{SnI}_{6}$ structure yield stronger electron-phonon coupling interactions that produce larger electron effective masses and reduced carrier mobilities relative to $\mathrm{Cs}_{2} \mathrm{SnI}_{6}$. Further, we discuss the results for $\mathrm{Rb}_{2} \mathrm{SnI}_{6}$ in the context of other vacancy-ordered double perovskite semiconductors, in order to demonstrate that the electron-phonon coupling characteristics can be predicted using the geometric perovskite tolerance factor. This study represents an important step in designing perovskite halide semiconductors with desired charge transport properties for optoelectronic applications.
\end{abstract}

\section{INTRODUCTION}

Inorganic perovskite halide-based materials have presented a paradigm shift in the search for new high performance semiconductors. The high amplitude lattice dynamics and low elastic moduli, yet excellent carrier dynamics and electronic behavior, suggest that elucidation of structure-dynamicsproperty relationships is required in order to fully understand these materials. ${ }^{1,2}$ The perovskite family is typified by the general formula $\mathrm{ABX}_{3}$, and the structure is formed by a network of corner sharing $\left[\mathrm{BX}_{6}\right]$ octahedra bridged by A-site cations in the cuboctahedral void. A hallmark of the perovskite family is cooperative octahedral tilting (static and dynamic) of the $\left[\mathrm{BX}_{6}\right]$ framework, which typically arises from a size mismatch between the A-site cation and the cuboctahedral void formed by 12 neighboring X-site anions, as predicted by the Goldschmidt tolerance factor, $t=\left(r_{\mathrm{A}}+r_{\mathrm{X}}\right) / \sqrt{2}\left(r_{\mathrm{B}}+r_{\mathrm{X}}\right) .^{3-5}$ Octahedral tilting distortions are well-known to influence the optoelectronic properties of perovskite halides, as the electronic states of the $\mathrm{B}$ - and $\mathrm{X}$-site ions dominate the conduction and valence band edges. ${ }^{6}$ Static octahedral tilting distortions affect the electronic dispersions of the $\mathrm{B}$ and $\mathrm{X}$ states through deviation in the $\mathrm{X}-\mathrm{B}-\mathrm{X}$ bond angle from $180^{\circ}$, yielding smaller carrier mobilities and higher resistivities. ${ }^{7}$ Changes in these bond angles also affect the relative energies of the conduction and valence band edges, resulting in a widening of the band gap with correspondingly larger octahedral tilting distortions. ${ }^{8-11}$

Received: April 13, 2018

Revised: May 23, 2018

Published: May 23, 2018 
Further, dynamic octahedral tilting of the $\left[\mathrm{BX}_{6}\right]$ framework has recently been implicated in the formation of polarons via electron-phonon coupling, which are hypothesized to protect carriers and screen crystallographic defects, thereby prolonging carrier excited state lifetimes and reducing carrier mobilities. $^{12-17}$

Vacancy-ordered double perovskites are a subset of the ordered double perovskite family with the general formula $\mathrm{A}_{2} \mathrm{BX}_{6}$. The structure is formed by a face-centered lattice of isolated $\left[\mathrm{BX}_{6}\right]$ octahedral units bridged by A-site cations. Similar to ordered double perovskites, vacancy-ordered double perovskites undergo successive octahedral tilting distortions according to the group-subgroup relationship $a^{0} a^{0} a^{0}(F m \overline{3} m)$ $\rightarrow a^{0} a^{0} c^{+}(P 4 / m n c) \rightarrow a^{+} b^{-} b^{-}\left(P 2_{1} / n\right),{ }^{18,19}$ and the proclivity of these materials to undergo octahedral tilting can be predicted by the "radius ratio", which is defined as the ratio of the A-site cation radius to the radius of the cuboctahedral site formed by the $\mathrm{X}_{12}$ cage. $^{20,21}$ As in conventional $\mathrm{ABX}_{3}$ perovskites, the electronic properties of vacancy-ordered double perovskites are directly dictated by the electronic states of the B- and X-site ions at the valence and conduction band edges. Close-packing of the halogen framework provides dispersive electronic states and a framework for mobile carriers, while the interaction of the $\mathrm{B}$-site cations with the coordinating X-site anions dictates the band positions, optical gaps, and tolerance to crystallographic defects. ${ }^{21-24}$ Although the electronic states of the A-site cations are typically far from the frontier electronic states in vacancyordered double perovskites, ${ }^{21}$ varying the identity of the A-site can indirectly influence characteristics such as carrier mobilities and band gap through changes in the dynamics of the surrounding inorganic framework. Our recent study of the vacancy-ordered double perovskites $\mathrm{A}_{2} \mathrm{SnI}_{6}$, where $\mathrm{A}=\mathrm{Cs}^{+}$, $\mathrm{CH}_{3} \mathrm{NH}_{3}^{+}$(methylammonium), and $\mathrm{CH}\left(\mathrm{NH}_{2}\right)_{2}^{+}$(formamidinium) revealed significant lattice anharmonicity in the hybrid compounds, resulting in more tightly bound polarons and subsequently reduced carrier mobilities across the series. ${ }^{25}$ Due to the large sizes and hydrogen bonding capabilities of the $\mathrm{CH}_{3} \mathrm{NH}_{3}^{+}$and $\mathrm{CH}\left(\mathrm{NH}_{2}\right)_{2}^{+}$cations, the anharmonic effects appear to manifest as random rotations of the isolated $\left[\mathrm{SnI}_{6}\right]$ octahedral units rather than through typical cooperative tilting motifs.

In this contribution, we present a study of the tin(IV) iodidebased vacancy-ordered double perovskite $\mathrm{Rb}_{2} \mathrm{SnI}_{6}$ and compare the observed structural and electronic properties to those of $\mathrm{Cs}_{2} \mathrm{SnI}_{6}$ and other $\mathrm{A}_{2} \mathrm{SnI}_{6}$ compounds. Both $\mathrm{Rb}_{2} \mathrm{SnI}_{6}$ and $\mathrm{Cs}_{2} \mathrm{SnI}_{6}$ exhibit native $n$-type conductivity, though $\mathrm{Rb}_{2} \mathrm{SnI}_{6}$ exhibits carrier mobilities that are reduced by $\sim 50$-fold relative to $\mathrm{Cs}_{2} \mathrm{SnI}_{6}$. Crystallographic analysis reveals that $\mathrm{Rb}_{2} \mathrm{SnI}_{6}$ crystallizes in the tetragonal modification of the vacancyordered double perovskite structure at room temperature due to cooperative tilting of the $\left[\mathrm{SnI}_{6}\right]$ octahedral units. Upon cooling, $\mathrm{Rb}_{2} \mathrm{SnI}_{6}$ undergoes further octahedral tilting to a lowersymmetry monoclinic structure. X-ray and neutron pair distribution function analysis reveal that the local coordination environment is best described by the monoclinic structure at all temperatures. This can be rationalized through bond valence sum analysis, which suggests that the $\mathrm{Rb}^{+}$ion coordination is optimized in the monoclinic structure. Calculation of the phonon dispersions indicate that, although the tetragonal structure is dynamically stable, the lowest-frequency optical phonon polarization corresponds to cooperative octahedral tilting that yields the monoclinic structure. This strongly implicates octahedral rotational dynamics in driving the structural phase transition. Density functional calculations reveal that cooperative octahedral tilting in $\mathrm{Rb}_{2} \mathrm{SnI}_{6}$ results in marginally smaller electron effective masses due to subtle changes in the close-packed iodide framework, which alone cannot account for the changes in carrier mobility observed experimentally. Rather, we find that the lower symmetry of $\mathrm{Rb}_{2} \mathrm{SnI}_{6}$ relative to $\mathrm{Cs}_{2} \mathrm{SnI}_{6}$ yields stronger electron-phonon coupling due to the larger number of nondegenerate lowfrequency phonons that contribute to the dielectric response of the lattice and produce more tightly bound polarons that reduce charge carrier mobilities. From these results we show that simple models such as bond valence sum and the perovskite tolerance factor serve as effective predictors for charge transport behavior in vacancy-ordered double perovskite semiconductors.

\section{METHODS AND MATERIALS}

Materials Synthesis. $\mathrm{SnI}_{4}{ }^{23,25}$ and $\mathrm{Cs}_{2} \mathrm{SnI}_{6}{ }^{23,26}$ were prepared by previously reported methods. $\mathrm{Rb}_{2} \mathrm{SnI}_{6}$ was synthesized via solution precipitation. To a $20 \mathrm{~mL}$ scintillation vial was added $0.2 \mathrm{~g}$ of $\mathrm{SnI}_{4}$, $10.0 \mathrm{~mL}$ of isopropanol, and $1.0 \mathrm{~mL}$ of hydriodic acid (57\% aq., unstabilized). The solution was heated to $T=60{ }^{\circ} \mathrm{C}$ while stirring to dissolve the entire mass of $\mathrm{SnI}_{4}$. In a separate scintillation vial was added a stoichiometric amount of $\mathrm{Rb}_{2} \mathrm{CO}_{3}$ to $2.0 \mathrm{~mL}$ of hydriodic acid and stirred to dissolve the solid. We note that it is important to minimize the length of time over which the $\mathrm{Rb}_{2} \mathrm{CO}_{3}$ is allowed to sit in the hydriodic acid, to prevent the formation of $\mathrm{RbI}_{3}$. Once the solids had dissolved in both solutions, the RbI solution was added all at once to the warm $\mathrm{SnI}_{4} /$ isopropanol solution. The scintillation vial was capped and stirred gently for $30 \mathrm{~min}$ to cool to room temperature. The solution was further cooled in an ice bath for an additional $30 \mathrm{~min}$ while stirring to induce precipitation. The black precipitate was collected via centrifugation and washed with a small amount of cold isopropanol. The product was dried in air at $T=60{ }^{\circ} \mathrm{C}$ for $24 \mathrm{~h}$. We note that unstabilized hydriodic acid is required for successful precipitation of the final product; use of $\mathrm{HI}$ containing the $\mathrm{H}_{3} \mathrm{PO}_{2}$ stabilizer typically results in precipitation of bright orange crystals, which are presumably due to precipitation of the $\mathrm{SnI}_{4}$ precursor.

Structural Characterization. High-resolution synchrotron powder X-ray diffraction data for $\mathrm{Rb}_{2} \mathrm{SnI}_{6}$ were collected from the diffractometer on beamline 11-BM-B at the Advanced Photon Source, Argonne National Laboratory, at $T=295 \mathrm{~K}$ and $T=100 \mathrm{~K}^{27}$ The data were analyzed with the Rietveld method implemented in GSAS/ EXPGUI. ${ }^{28,29}$ VESTA was used to visualize and render all crystal structures presented in this publication. ${ }^{30}$

Synchrotron X-ray scattering data suitable for pair distribution function (PDF) analysis were collected at beamline 11-ID-B at the Advanced Photon Source, Argonne National Laboratory, using $86 \mathrm{keV}$ photons and sample-detector distance of $25 \mathrm{~cm}$. Powdered samples of $\mathrm{Rb}_{2} \mathrm{SnI}_{6}$ were loaded into polyimide capillaries and measured in transmission geometry at room temperature using a PerkinElmer amorphous silicon image plate detector. ${ }^{31}$ The program Fit $2 \mathrm{D}^{32}$ was used to calibrate the sample to detector distance and detector alignment with data from a $\mathrm{CeO}_{2}$ powder standard. Raw scattering data was integrated into Q-space spectra, applying a mask and polarization correction during integration. Experimental PDFs were extracted using PDFgetX2 $2^{33}$ and analyzed using PDFgui. ${ }^{34}$ The normalized total scattering pattern, $S(Q)$, was produced in PDFgetX2 by subtracting polyimide container scattering, utilizing the appropriate sample composition, and applying standard corrections for the area detector setup. ${ }^{31}$ The pair distribution function pattern, $G(r)$, was calculated via sine Fourier transformation of the total scattering data utilizing a maximum $Q$ of $23.8 \AA^{-1}$. Values of $Q_{\text {damp }}=0.034127 \AA^{-1}$ and $Q_{\text {broad }}=0.021102 \AA^{-1}$ were extracted from refinement of a $\mathrm{TiO}_{2}$ anatase standard in PDFgui and used for further refinement.

Neutron scattering measurements were performed on the NOMAD instrument at the Spallation Neutron Source, Oak Ridge National Laboratory. A powdered sample of $\mathrm{Rb}_{2} \mathrm{SnI}_{6}$ was loaded into a $6 \mathrm{~mm}$ 
vanadium sample can and sealed under a $\mathrm{He}$ atmosphere. Total scattering data were collected at $T=2,10,150,250$, and $300 \mathrm{~K}$ in the cryostat sample environment. Data were normalized against scattering data collected for a vanadium rod, and background scattering from the vanadium can was subtracted. Total scattering data of $\mathrm{Cs}_{2} \mathrm{SnI}_{6}$ at $T=$ $10 \mathrm{~K}$ were collected on a powdered sample of $\mathrm{Cs}_{2} \mathrm{SnI}_{6}$ sealed into a 6 $\mathrm{mm}$ vanadium sample can under a $\mathrm{He}$ atmosphere utilizing the cryostat sample environment. Data were normalized against scattering data collected for a vanadium rod, and background scattering from the vanadium can was subtracted. Total scattering data of $\mathrm{Cs}_{2} \mathrm{SnI}_{6}$ at $T=$ $90 \mathrm{~K}$ and $T=300 \mathrm{~K}$ were collected on powdered samples of $\mathrm{Cs}_{2} \mathrm{SnI}_{6}$ sealed into a quartz capillary (capillary diameter $=3.0 \mathrm{~mm}$ ) in the multisample changer. Data were normalized against scattering data collected for an empty glass capillary, and background scattering from the empty capillary was subtracted.

For all neutron total scattering experiments, the data were merged to the total scattering structure function using the IDL codes developed for the NOMAD instrument. ${ }^{35}$ The pair distribution function was then produced through the sine Fourier transform of the total scattering structure function utilizing $Q_{\max }=31.4 \AA^{-1}$. For $\mathrm{Rb}_{2} \mathrm{SnI}_{6}$ at all temperatures, values of $Q_{\text {damp }}=0.0245 \AA^{-1}$ and $Q_{\text {broad }}=$ $0.0196 \AA^{-1}$ were extracted from refinement of a silicon standard in PDFgui. For $\mathrm{Cs}_{2} \mathrm{SnI}_{6}$ at $T=90 \mathrm{~K}$ and $T=300 \mathrm{~K}$, values of $Q_{\text {damp }}=$ $0.0201 \AA^{-1}$ and $Q_{\text {broad }}=0.0196 \AA^{-1}$ were extracted from refinement of a diamond standard in PDFgui. For $\mathrm{Cs}_{2} \mathrm{SnI}_{6}$ at $T=10 \mathrm{~K}$, values of $Q_{\text {damp }}=0.01766 \AA^{-1}$ and $Q_{\text {broad }}=0.01918 \AA^{-1}$ were extracted from refinement of a silicon standard. Analysis of the nPDFs was performed using PDFgui.

Optical and Electronic Properties. UV-visible diffuse reflectance spectroscopy was performed on powdered samples of $\mathrm{Rb}_{2} \mathrm{SnI}_{6}$ diluted to 15 wt \% in $\mathrm{BaSO}_{4}$, using $\mathrm{BaSO}_{4}$ as a baseline. Spectra were acquired using a Thermo Nicolet Evolution 300 spectrophotometer with a Praying Mantis mirror setup from $\lambda=600-1000 \mathrm{~nm}$ at a scan rate of $240 \mathrm{~nm} / \mathrm{min}$.

Electrical resistance measurements were performed on cold-pressed polycrystalline pellets of $\mathrm{Rb}_{2} \mathrm{SnI}_{6}$ using a Physical Properties Measurement System (Quantum Design, Inc.). The measurements were collected in a linear 4-probe configuration using Pt wires and Agpaste contacts. Similar results were obtained when Au paste was used. Hall measurements were collected on a cold-pressed polycrystalline pellet in the Van der Pauw configuration at $T=300 \mathrm{~K}$ with Pt wires contacted to the edge of the cylindrical pellet using $\mathrm{Ag}$ paste. 4-probe sample geometry: $l=0.35(1) \mathrm{cm}, w=0.35(1) \mathrm{cm}, h=0.05(1) \mathrm{cm}$. Hall probe sample thickness: $t=0.08(1) \mathrm{cm}$.

Density Functional Theory Calculations. Density functional theory calculations were performed using the Vienna $A b$ initio Simulation Package (VASP). ${ }^{36-39}$ A plane-wave basis set was employed, with the interactions between core and valence electrons described using the Projector Augmented Wave (PAW) method. ${ }^{40}$ This study employed two functionals: PBEsol, ${ }^{41}$ a version of the Perdew, Burke and Erzorhof (PBE) functional ${ }^{42}$ revised for solids, and the hybrid functional HSE06 which combines $75 \%$ exchange and 100\% of the correlation energies from PBE, together with $25 \%$ exact Hartree-Fock (HF) exchange at short-range. ${ }^{43}$ PBEsol has been shown to reproduce the structural and vibrational properties of many compounds containing weakly dispersive interactions, such as in the vacancy-ordered double perovskites ${ }^{25}$ and other layered halide systems. ${ }^{44,45}$ The band structures were calculated along a reciprocal space path defined between all high-symmetry points in the Brillouin zone, as detailed by Bradley and Cracknell. ${ }^{4}$ For density of states, band structure, and high-frequency dielectric response calculations, special attention was paid to capturing the effects of electron-electron interactions through use of the HSE06 hybrid functional. ${ }^{47,48}$ Furthermore, the relativistic effects seen in Sn and I were taken into account through use of scalar relativistic pseudopotentials and explicit treatment of spin-orbit coupling effects. ${ }^{49}$ This combination of HSE06+SOC has been shown to provide an accurate description of the electronic structure of many metal-halide containing semiconductors. ${ }^{50,51}$ A $k$-point sampling mesh of $\Gamma$ centered $3 \times 3 \times 2$ and plane wave energy cutoff of $350 \mathrm{eV}$ were found to converge the total energy to $1 \mathrm{meV} /$ atom for all systems studied. The structures were relaxed using HSE06 until the forces on all atoms totaled less than 0.01 $\mathrm{eV} \AA^{-1}$. The HSE06 optimized crystal structures can be found online in a public repository. ${ }^{52}$

The ionic contribution to the dielectric constants were calculated using the PBEsol functional within density functional perturbation theory $(\mathrm{DFPT}){ }^{53}$ with a denser $6 \times 6 \times 4 \Gamma$-centered $k$-point mesh necessary to reach convergence. The phonon band structure was calculated using the finite-displacement method in a $3 \times 3 \times 2(324$ atom) supercell using a $1 \times 1 \times 1 k$-point mesh. The DFPT calculations were performed on structures relaxed using PBEsol, due to the high computational expense of performing DFPT calculations using hybrid DFT. The high-frequency real and imaginary dielectric functions were calculated from the optical transition matrix elements within the transversal approximation, ${ }^{54}$ obtained at a denser $6 \times 6 \times 3$ $\Gamma$-centered $k$-point mesh. Band alignments were performed using a vacuum-slab model ( $35 \AA$ slab, $35 \AA$ vacuum) with a (101) surface. The corresponding electrostatic potential was averaged along the $c$ direction using the MacroDensity package, ${ }^{55}$ with the energy of the potential at the plateau used as the external vacuum level. Slab calculations were performed using the HSE06 functional, with a correction for the valence band energy and band gap taken from the HSE06+SOC calculated bulk.

Polaron mobilities were calculated within a temperature-dependent Feynman model, as implemented in the codes produced by Frost. ${ }^{56} \mathrm{~A}$ full definition of the methodology is described elsewhere in the literature. ${ }^{57}$ Crucially, the electron-phonon coupling is approximated without empirical parameters, using a highly idealized model. ${ }^{58-60}$ The band structure is represented only as the effective mass approximation, with the physical response of the lattice given by the optical and static dielectric constants and an effective phonon-response frequency. This method has recently been applied to calculate the electron mobilities in the hybrid perovskites ${ }^{57}$ and other vacancy-ordered double perovskites. $^{25}$

\section{RESULTS}

Structural Characterization. Rubidium tin(IV) iodide crystallizes in the tetragonal modification of the vacancyordered double perovskite structure (space group $P 4 / m n c$ ) at room temperature, in contrast to a previous report of a cubic structure. ${ }^{61}$ The structure is characterized by in-phase cooperative octahedral rotations about the $c$-axis by $\sim 11^{\circ}$ $\left(a^{0} a^{0} c^{+}\right.$in Glazer notation) relative to the cubic aristotype, as shown in Figure 1a,b. The tetragonal structural model was refined against high-resolution synchrotron powder X-ray diffraction (SXRD) data shown in Figure 2a,b.

Upon cooling to $T=100 \mathrm{~K}, \mathrm{Rb}_{2} \mathrm{SnI}_{6}$ undergoes a phase transition to monoclinic symmetry (space group $P 2_{1} / n$ ), characterized by out-of-phase tilting of the octahedra in the $a b$ plane by $\sim 5^{\circ}\left(a^{-} a^{-} c^{+}\right)$, in addition to the $11^{\circ}$ tilt about the $c$-axis. The transition is evidenced by splitting of the (202) reflection in the SXRD patterns shown in Figure $2 c, d$ and is consistent with the series of symmetry-lowering phase transitions commonly observed in other members of the vacancy-ordered double perovskite family and with those predicted for ordered double perovskites. ${ }^{18,62,63}$ Structural and statistical parameters generated from Rietveld refinements of the tetragonal and monoclinic structural models against the SXRD data can be found in Table 1, and crystallographic information files are included as Supporting Information.

Temperature-dependent neutron scattering data collected on the NOMAD beamline at the Spallation Neutron Source, Oak Ridge National Laboratory, provide insight into the phase behavior of $\mathrm{Rb}_{2} \mathrm{SnI}_{6}$. Low-fidelity scans were collected continuously upon cooling from $T \sim 298 \mathrm{~K}$ to $T \sim 10 \mathrm{~K}$. The reflections shift to higher $Q$ with decreasing temperature 


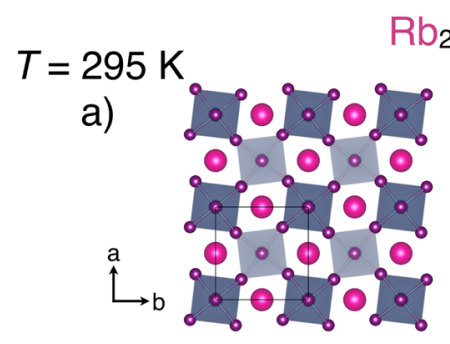

$\mathrm{Rb}_{2} \mathrm{Snl}_{6}$
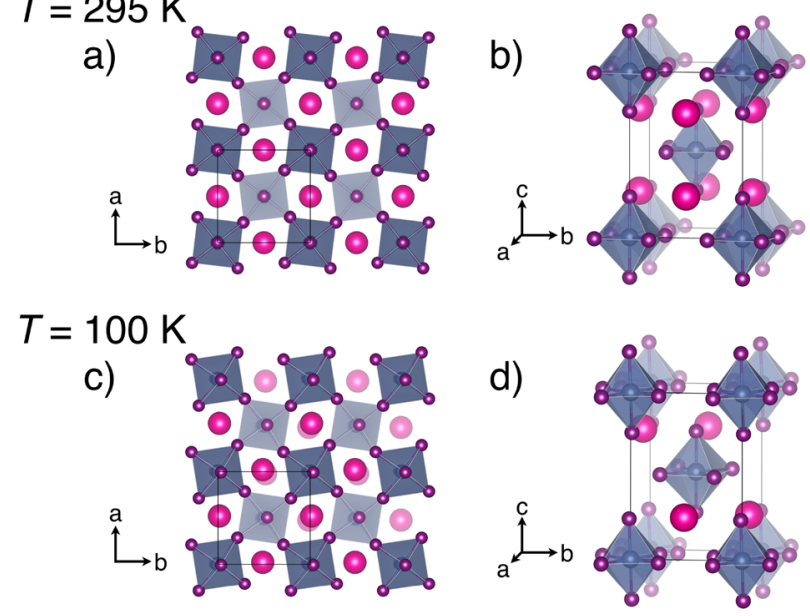

Figure 1. Crystal structures of $\mathrm{Rb}_{2} \mathrm{SnI}_{6}$ at $T=295 \mathrm{~K}$ and $T=100 \mathrm{~K}$. In (a) and (c), the structures are projected down the $c$-axis to highlight the octahedral tilting and rotation, while unit cell descriptions are shown in (b) and (d). Rubidium atoms are shown in pink, tin atoms in blue, and iodine atoms in purple.

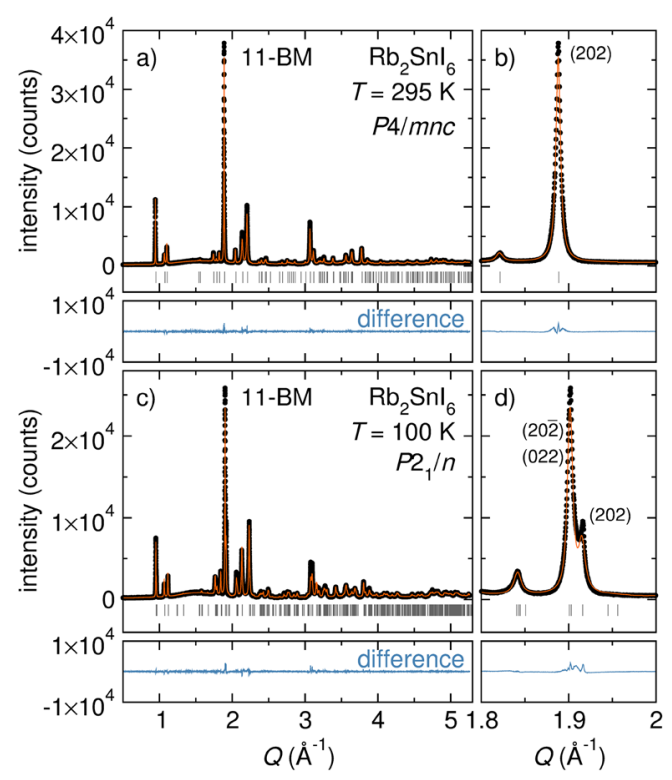

Figure 2. Rietveld refinements of the tetragonal and monoclinic structural models of $\mathrm{Rb}_{2} \mathrm{SnI}_{6}$ against high resolution synchrotron powder X-ray diffraction patterns collected at $T=295 \mathrm{~K}(\mathrm{a}, \mathrm{b})$ and $T$ $=100 \mathrm{~K}(\mathrm{c}, \mathrm{d})$ on the high-resolution 11-BM diffractometer. Data are shown as black circles, the fit is the orange line, and the difference is shown in blue. Plots (b) and (d) highlight the splitting of the most intense reflection (202) at $Q \sim 1.9 \AA^{-1}$ upon cooling through the phase transition.

consistent with thermal contraction of the lattice upon cooling and show subtle evidence of a phase transition near $T \sim 189 \mathrm{~K}$ (Figure S1, as elaborated in the SI).

Electronic and Optical Properties. Electrical resistivity measurements of $\mathrm{Rb}_{2} \mathrm{SnI}_{6}$ performed in a 4-probe configuration reveal semiconducting behavior, as evidenced by increasing resistivity upon cooling shown in Figure 3. At $T=300 \mathrm{~K}$, $\mathrm{Rb}_{2} \mathrm{SnI}_{6}$ exhibits a bulk resistivity of $\rho=\sim 5 \times 10^{5} \Omega \cdot \mathrm{cm}$, which is approximately 5 orders of magnitude larger than that reported for $\mathrm{Cs}_{2} \mathrm{SnI}_{6}(\rho=12 \Omega \cdot \mathrm{cm}) .{ }^{23}$ Hall effect measure-
Table 1. Structural Parameters and Refinement Statistics for $\mathrm{Rb}_{2} \mathrm{SnI}_{6}$ at $T=295 \mathrm{~K}$ and $T=100 \mathrm{~K}$

\begin{tabular}{lll} 
& \multicolumn{1}{c}{$295 \mathrm{~K}$} & \multicolumn{1}{c}{$100 \mathrm{~K}$} \\
crystal system & tetragonal & monoclinic \\
space group & $P 4 / m n c$ & $P 2_{1} / n$ \\
$a(\AA)$ & $8.07056(6)$ & $7.9426(1)$ \\
$b(\AA)$ & $8.07056(6)$ & $7.9758(9)$ \\
$c(\AA)$ & $11.7650(1)$ & $11.7876(2)$ \\
$\alpha(\mathrm{deg})$ & 90 & 90 \\
$\beta(\mathrm{deg})$ & 90 & $90.484(1)$ \\
$\gamma(\mathrm{deg})$ & 90 & 90 \\
$U_{\text {iso }}(\mathrm{Rb})\left(\AA^{2}\right)$ & $0.0564(8)$ & $0.0245(7)$ \\
$U_{\text {iso }}(\mathrm{Sn})\left(\AA^{2}\right)$ & $0.0245(6)$ & $0.0194(7)$ \\
$U_{\text {iso }}(\mathrm{I} 1)\left(\AA^{2}\right)$ & $0.0587(7)$ & $0.0296(5)$ \\
$U_{\text {iso }}(\mathrm{I} 2)\left(\AA^{2}\right)$ & $0.0521(4)$ & $0.0262(5)$ \\
$U_{\text {iso }}(\mathrm{I} 3)\left(\AA^{2}\right)$ & - & $0.0219(5)$ \\
red. $\chi^{2}$ & 5.373 & 9.046 \\
wR & $8.41 \%$ & $10.92 \%$ \\
\hline
\end{tabular}

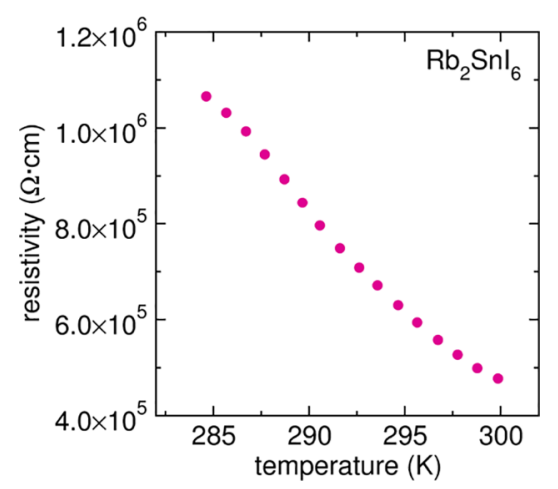

Figure 3. Temperature-dependent resistivity data of rubidium tin(IV) iodide collected using a 4-probe configuration with $\mathrm{Pt}$ wires and $\mathrm{Ag}$ paste.

ments at $T=300 \mathrm{~K}$ reveal that $\mathrm{Rb}_{2} \mathrm{SnI}_{6}$ exhibits native $n$-type conductivity, consistent with the formation of iodine vacancy defect states that form as shallow donors to the conduction band, as in other tin(IV)-iodide based vacancy-ordered perovskites. ${ }^{22,23,25}$ The higher resistivity of $\mathrm{Rb}_{2} \mathrm{SnI}_{6}$ compared with $\mathrm{Cs}_{2} \mathrm{SnI}_{6}$ arises from a reduction in both carrier concentration and carrier mobility. From Hall effect measurements, $\mathrm{Rb}_{2} \mathrm{SnI}_{6}$ exhibits a carrier concentration on the order of $n_{e} \sim 10^{12} \mathrm{~cm}^{-3}$ and a carrier mobility of $\mu_{e} \sim 0.22(3) \mathrm{cm}^{2} \mathrm{~V}^{-1}$ $\mathrm{s}^{-1}$, which are reduced by factors of $\sim 10^{4}$ and $\sim 50$ respectively compared with $n_{e} \sim 5(1) \times 10^{16} \mathrm{~cm}^{-3}$ and $\mu_{e} \sim 8.6(5) \mathrm{cm}^{2} \mathrm{~V}^{-1}$ $\mathrm{s}^{-1}$ reported for $\mathrm{Cs}_{2} \mathrm{SnI}_{6}{ }^{23}$

Analysis of UV-visible diffuse reflectance spectroscopy data for $\mathrm{Rb}_{2} \mathrm{SnI}_{6}$ yields an optical gap of $1.32(2) \mathrm{eV}$, consistent with the black color of the compound. The optical gap was extracted by converting the raw reflectance data to pseudoabsorbance, $F(R)$, using the Kubelka-Munk function. The optical gap was determined by extrapolating the linear absorption onset region to zero absorbance, as shown in Figure 4. The optical gap of $1.32(2) \mathrm{eV}$ is slightly larger than the value of $1.23(3) \mathrm{eV}$ determined for $\mathrm{Cs}_{2} \mathrm{SnI}_{6}$ by the same method. ${ }^{23,25}$

Local Coordination Environment. X-ray and neutron pair distribution function (XPDF, nPDF) analyses were employed to correlate changes in the local coordination environment with the observed structural and electronic behavior. The XPDF of rubidium $\operatorname{tin}(\mathrm{IV})$ iodide at $T=300 \mathrm{~K}$ is shown in Figure 5. The 


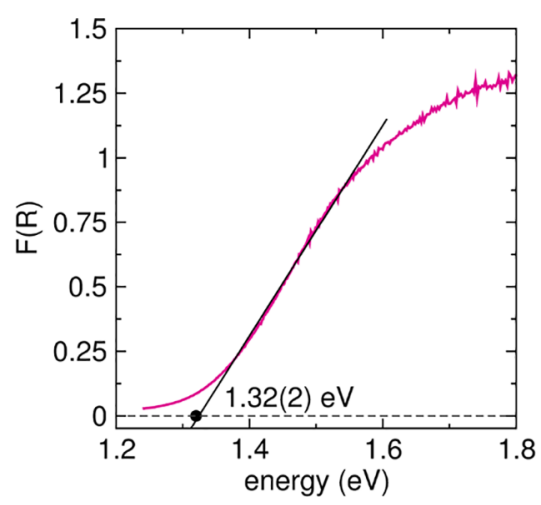

Figure 4. UV-visible diffuse reflectance spectrum for $\mathrm{Rb}_{2} \mathrm{SnI}_{6}$. The data were converted to pseudoabsorbance via the Kubelka-Munk transform, and the optical gap was determined by extrapolating the onset region to zero absorbance, as shown by the black fit. The pink line is the transformed data, the fit is the black line, and zero absorbance is shown as the gray dashed line. The intersection point of the fit and zero absorbance is shown by the black dot.

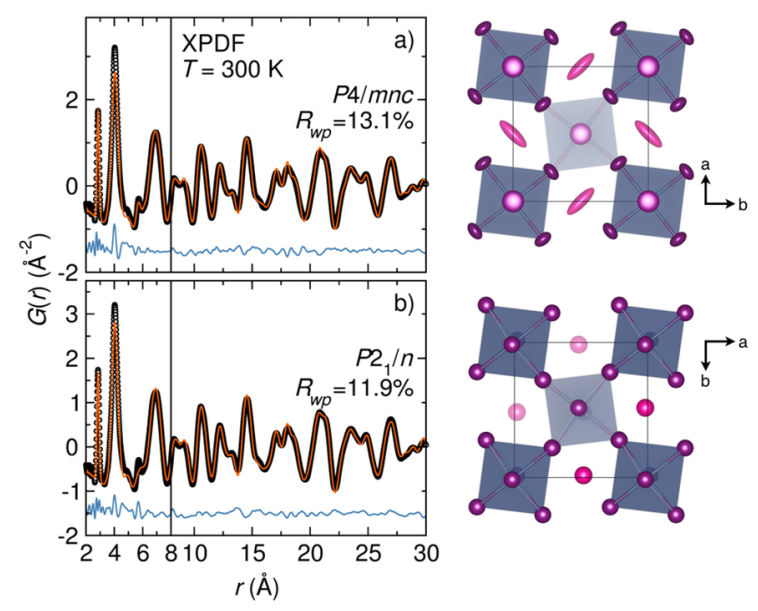

Figure 5. X-ray pair distribution function of $\mathrm{Rb}_{2} \mathrm{SnI}_{6}$ fit to the (a) tetragonal $(P 4 / m n c)$ and $(\mathrm{b})$ monoclinic $\left(P 2_{1} / n\right)$ structural models. In (a), the XPDF is fit to the tetragonal $(P 4 / m n c)$ model using anisotropic atomic displacement parameters for iodine and rubidium ions and in (b) the XPDF is fit to the monoclinic $\left(P 2_{1} / n\right)$ structural model using isotropic displacement parameters for all atoms. The thermal ellispoids in the corresponding structural models are shown at $95 \%$ probability.

local coordination environment of $\mathrm{Rb}_{2} \mathrm{SnI}_{6}$ is moderately welldescribed by the tetragonal structural model observed in the powder X-ray diffraction data. Modeling the data with this structural model requires the inclusion of large, anisotropic atomic displacement parameters (ADPs) for the iodine and rubidium atoms, as represented by the thermal ellipsoids on the structural models shown adjacent to the fit. Yet, the model still does not capture the shape of the next-nearest neighbor I-I pair correlation at $r \sim 4 \AA$. The large ADPs required to provide an adequate fit to the data suggest the presence of disorder (static or dynamic) of the iodine and rubidium atoms within the structure. The fit to the XPDF is significantly improved, both statistically and visually, with the use of the lowtemperature monoclinic structural model determined from the $T=100 \mathrm{~K}$ SXRD data, as shown in Figure 5b. The addition of out-of-phase octahedral tilting into the $a b$ plane permitted by the lower symmetry of the monoclinic structure results in significantly smaller isotropic ADPs for all atoms, as shown by the thermal ellipsoids in the corresponding structural model.

This analysis indicates that the local coordination environment at $T=300 \mathrm{~K}$ is best modeled by the monoclinic structural modification, despite the higher-symmetry tetragonal structure observed by SXRD. This observation is consistent with previous reports of other perovskite halide materials, in which dynamic and cooperative octahedral rotations manifest as a lower-symmetry instantaneous structure ${ }^{64}$ in the local coordination environment, yet they average to higher symmetry structures observed by diffraction. ${ }^{94,16,25,65-68}$ The large thermal ellipsoids observed for both iodine and rubidium atoms implies that there is strong coupling between octahedral tilting and $\mathrm{Rb}^{+}$displacements, as has been previously presented. ${ }^{5}$

Temperature-dependent neutron pair distribution function analysis (nPDF) was performed to probe changes in the local coordination environment of $\mathrm{Cs}_{2} \mathrm{SnI}_{6}$ and $\mathrm{Rb}_{2} \mathrm{SnI}_{6}$ as a function of temperature. At all temperatures, the local coordination environment of $\mathrm{Cs}_{2} \mathrm{SnI}_{6}$ is well-described by the cubic structural model, with no evidence of symmetry-lowering phase transitions, consistent with the corresponding neutron diffraction patterns shown in Figure $6 \mathrm{~b}$ and with our previous report. $^{23}$ We note that a slight asymmetry of the next-nearest neighbor I-I pair correlation at $r \sim 4 \AA$ is observed at $T=300$ $\mathrm{K}$, which has been previously attributed to anharmonic lattice dynamics. $^{25}$ The fits of the cubic structural model to the nPDF are shown in Figure 6a, and Rietveld refinements of the cubic structural model against the corresponding diffraction data are shown in Figure 6b.

Temperature-dependent nPDFs of $\mathrm{Rb}_{2} \mathrm{SnI}_{6}$ provide further insight into the structural behavior observed by SXRD and XPDF. For $T>150 \mathrm{~K}$, the $\mathrm{nPDF}$ can be modeled with the tetragonal structural model with the inclusion of large, anisotropic ADPs for the iodine atoms or with the monoclinic structural model with isotropic ADPs, consistent with analysis of the XPDF shown in Figure 5. Upon cooling to $T=150 \mathrm{~K}$, a low- $r$ shoulder to the next-nearest neighbor I-I pair correlation at $r=4 \AA$ appears, which is only captured by the monoclinic structural model, as shown in Figure 7a. The appearance of this shoulder results in a poor fit to the nPDF when modeling with the higher-symmetry tetragonal structure. Rietveld refinements of the corresponding neutron diffraction data are shown Figure 7b. Data collected at temperatures above $T=150 \mathrm{~K}$ are modeled with the tetragonal $P 4 / m n c$ structure, while data collected at $T=150 \mathrm{~K}$ and below are modeled by the $P 2_{1} / n$ structure.

Electronic Structure Calculations. Density functional calculations were performed to gain insight into the optical and electronic properties of rubidium tin(IV) iodide. The orbitalprojected band structures of $\mathrm{Rb}_{2} \mathrm{SnI}_{6}$ in the $P 4 / m n c$ and $P 2_{1} / n$ structures, calculated using HSE06+SOC, are shown in Figure 8. Both symmetries yield direct band gaps, with fundamental band gaps of 1.13 and $1.32 \mathrm{eV}$ occurring at the $\Gamma$ point (Table 2). Spin-orbit coupling was found to play a role on the size of the band gaps, with a band gap renormalization of 0.19 and $0.21 \mathrm{eV}$ seen, respectively, mainly though raising of the valence band maximum. For both compounds, the orbital projections reveal the upper valence band to be dominated by I $5 p$ states, with the conduction band minimum derived from hybridization between $\mathrm{Sn} 5 s$ and I $5 p$ states, in agreement with other $\mathrm{A}_{2} \mathrm{SnI}_{6}$ perovskite materials. ${ }^{23,25}$ We note that the calculated fundamental band gap of the $P 4 / m n c$ phase is significantly 
a)

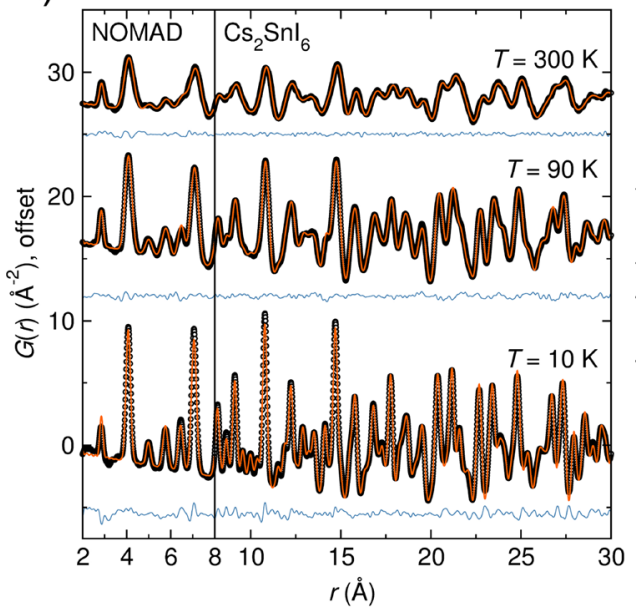

b)

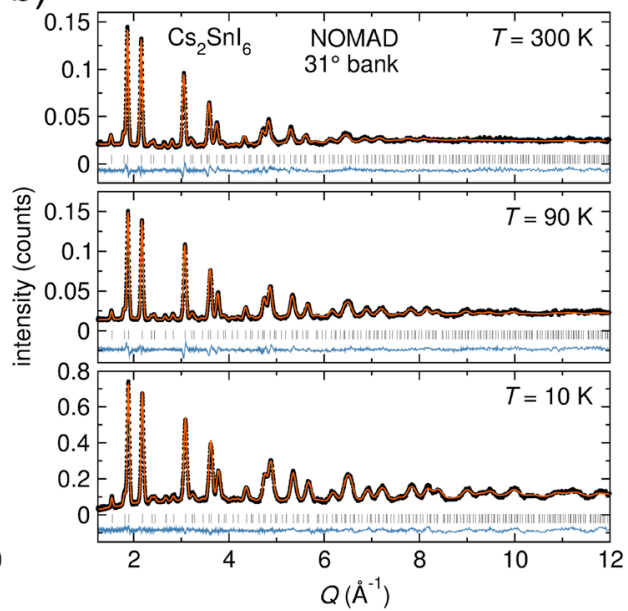

Figure 6. (a) Temperature-dependent neutron pair distribution function analysis (nPDF) of $\mathrm{Cs}_{2} \mathrm{SnI}_{6}$ extracted from neutron total scattering collected on NOMAD. The $x$-axis is split to highlight the low-r pair correlations. Rietveld refinements of the corresponding neutron diffraction patterns collected from bank 2 ( $31^{\circ}$ bank) of NOMAD are shown in (b). Both the nPDF and neutron diffraction data are modeled by the cubic $(F m \overline{3} m)$ structural model at all temperatures. Black circles are the data, the orange lines are the fits, and the blue lines are difference curves. In $(\mathrm{b})$, the gray tick marks indicate the positions of predicted reflections from the $F m \overline{3} m$ structure.

a)

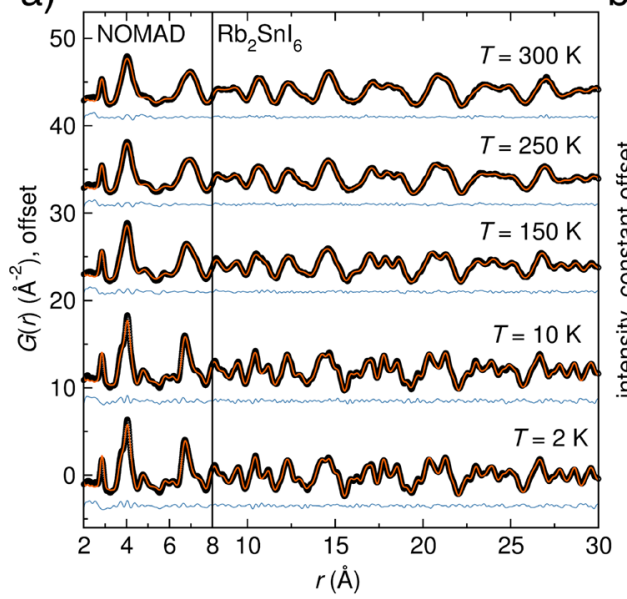

b)

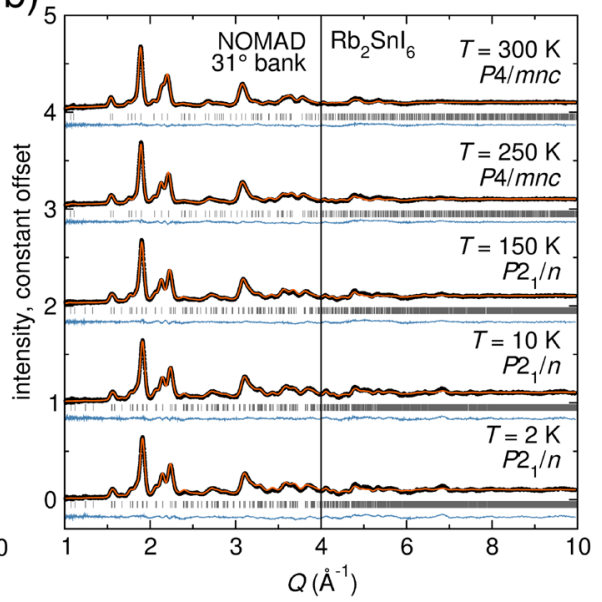

Figure 7. (a) Temperature-dependent neutron pair distribution function analysis (nPDF) of $\mathrm{Rb}_{2} \mathrm{SnI}_{6}$ extracted from neutron total scattering collected on NOMAD. The nPDFs are best modeled by the monoclinic $\left(P 2_{1} / n\right)$ structure at all temperatures. The $x$-axis is split to highlight the low$r$ pair correlations, and the data are offset vertically for clarity. Rietveld refinements of the neutron diffraction data from bank 2 ( $31^{\circ}$ bank) of NOMAD are shown in (b). For $T>150 \mathrm{~K}$, the data are modeled with the tetragonal $(P 4 / m n c)$ structure. For $T \leq 150 \mathrm{~K}$, the data are modeled by the monoclinic $\left(P 2_{1} / n\right)$ structure. Black circles are the data, the orange lines are the fits, and the blue lines are difference curves. In (b), the gray tick marks indicate the positions of predicted reflections from the $P 4 / m n c$ and $P 2_{1} / n$ structures. The $x$-axis is split to highlight the lower- $Q$ reflections, and the data are offset vertically for clarity.
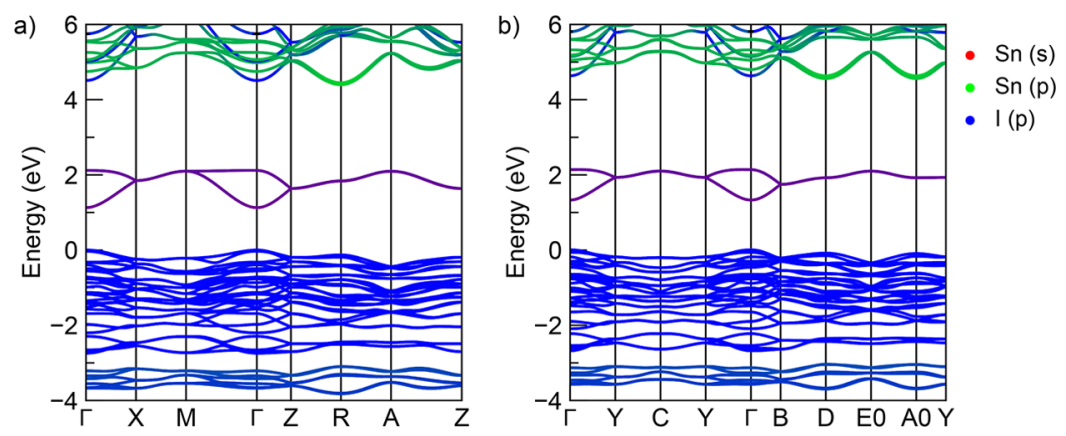

Figure 8. Band structures calculated using HSE06+SOC for the (a) $P 4 / m n c$ and (b) $P 2_{1} / n$ phases of $\mathrm{Rb}_{2} \mathrm{SnI}_{6}$. The color of the band indicates the orbital contribution to that band, with $S n 5 s$, Sn $5 p$, and I $5 p$ represented by red, green, and blue, respectively. The resulting color of the bands is obtained by mixing each color in proportion to the orbital contributions. The valence band maximum is set to $0 \mathrm{eV}$ in all cases. 
Table 2. Band Gaps $\left(E_{\mathrm{g}}\right)$, Conduction Bandwidths $\left(\Delta \varepsilon_{\mathrm{CB}}\right)$, and Charge Carrier Effective Masses $\left(m^{*}\right)$, Calculated Using HSE06+SOC, for the P4/mnc and $P 2_{1} / n$ Phases of $\mathrm{Rb}_{2} \mathrm{SnI}_{6}{ }^{a}$

$\begin{array}{lcccc}\text { phase } & E_{\mathrm{g}} & \Delta \varepsilon_{\mathrm{CB}} & m_{e}^{*} & m_{h}^{*} \\ P 4 / m n c & 1.13 & 0.99 & 0.39 & 0.98 \\ P 2_{1} / n & 1.32 & 0.81 & 0.44 & 1.07\end{array}$

${ }^{a}$ Band gaps and widths are provided in $\mathrm{eV}$, and effective masses are given in units of the bare electron mass, $m_{0}$.

smaller than the optical band gap measured in experiment (1.32 $\mathrm{eV})$. Analysis of the transition dipole moments reveals that, consistent with other $\mathrm{A}_{2} \mathrm{SnI}_{6}$ materials, ${ }^{23}$ the fundamental band gap is dipole disallowed in both symmetries. Instead, the optical band gap results from transitions originating from the bands $\sim 0.30 \mathrm{eV}$ below the valence band maximum.

The carrier effective masses, calculated based on parabolic fits of the band edges in three directions around the $\Gamma$ point, indicate electrons will be relatively mobile, with masses of 0.39 $m_{0}$ and $0.44 m_{0}$ seen for the $P 4 / m n c$ and $P 2_{1} / n$ phases, respectively. These are marginally larger than the electron effective masses in $\mathrm{Cs}_{2} \mathrm{SnI}_{6}\left(m_{e}^{*}=0.25 m_{0}\right)$, ${ }^{25}$ likely due to deviations in the close-packed iodine sublattice from octahedral tilting. These changes further manifest as a reduction in the conduction bandwidth, $\Delta \varepsilon_{\mathrm{CB}}$, from $1.39 \mathrm{eV}$ seen in $\mathrm{Cs}_{2} \mathrm{SnI}_{6}$, to 0.99 and $0.81 \mathrm{eV}$, for the $P 4 / m n c$ and $P 2_{1} / n$ phases, respectively.

DFT calculations of isolated slab structures surrounded by vacuum reveal the band gap differences between the two phases of $\mathrm{Rb}_{2} \mathrm{SnI}_{6}$ are driven by changes in the position of both the valence band maximum and the conduction band minimum. For the P4/mnc phase, the HSE06+SOC calculated ionization potential and electron affinity are 5.82 and $4.70 \mathrm{eV}$, respectively (Figure 9). Moving to the low temperature $P 2_{1} / n$ phase, the

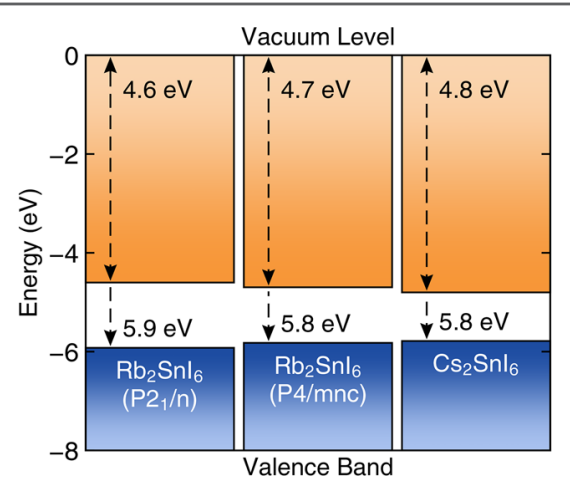

Figure 9. Calculated band alignment (HSE06+SOC) of the $P 4 / m n c$ and $P 2_{1} / n$ structured phases of $\mathrm{Rb}_{2} \mathrm{SnI}_{6}$ relative to those of $\mathrm{Cs}_{2} \mathrm{SnI}_{6}$.

ionization potential is deeper relative to the vacuum level (5.92 $\mathrm{eV}$ ), whereas the electron affinity becomes more shallow (4.60 $\mathrm{eV}$ ). Again, these changes can likely be attributed to subtle distortions of the close-packed iodine sublattice and the reduction in bandwidth of both the upper valence and the lower conduction bands. We note that the band alignments are close to those calculated for $\mathrm{Cs}_{2} \mathrm{SnI}_{6}$, which shows an ionization potential and electron affinity of 5.79 and $4.80 \mathrm{eV}$, respectively; this is not unexpected due to the similar orbital composition of the valence and conduction bands in these materials.

To further investigate the carrier mobilities in $\mathrm{Rb}_{2} \mathrm{SnI}_{6}$, we have calculated the limits of electron mobility within a temperature-dependent Feynman polaron transport model. This method has recently been applied to the cubic hybrid perovskites ${ }^{57}$ and other members of the $\mathrm{A}_{2} \mathrm{SnI}_{6}$ series. $^{25}$ All required parameters were calculated $a b$ initio and are provided in Table 3. The electron effective masses necessary for the

\section{Table 3. Parameters of the Feynman Polaron Model ${ }^{a}$}

$\begin{array}{cccc}\text { space group } & \varepsilon_{\infty} & \varepsilon_{S} & f \\ P 4 / m n c & 3.61 & 8.38 & 4.01 \\ P 2_{1} / n & 3.64 & 8.48 & 4.32\end{array}$

${ }^{a}$ High frequency $\left(\varepsilon_{\infty}\right)$ and static $\left(\varepsilon_{S}\right)$ dielectric constants are given in units of the permittivity of free space $\left(\varepsilon_{0}\right)$. Frequency $(f)$ is in THz.

calculations, $m_{e}^{*}$, are provided in Table 2 . Within this model, the extent of electron-phonon coupling $(\alpha)$ is used to evaluate the polaron mobility $\left(\mu_{e}^{H}\right.$, calculated within the Hellwarth model), ${ }^{58}$ along with the associated phonon-drag massrenormalization $\left(m_{r}^{*}\right)$ and relaxation time $(\tau)$. The Hellwarth electron mobilities, $\mu_{e}^{H}$, at $T=300 \mathrm{~K}$ are presented in Table 4 .

Table 4. Hellwarth Electron Mobilities at $T=300 \mathrm{~K}\left(\mu_{e}^{H}, \mathrm{~cm}^{2}\right.$ $\left.\mathrm{V}^{-1} \mathbf{s}^{-1}\right)$, Electron-Phonon Coupling $(\alpha)$, Effective Mass Renormalization $\left(m_{r}^{*}\right.$, units of $\left.m^{*}\right)$, and Polaron Relaxation Time $(\tau, \mathrm{ps})$, Calculated within a Temperature-Dependent Polaron Model, for the $\mathrm{P4} / \mathrm{mnc}$ and $\mathrm{P2} / \mathrm{n}$ Phases of $\mathrm{Rb}_{2} \mathrm{SnI}_{6}$

\begin{tabular}{ccccc} 
space group & $\mu_{e}^{H}$ & $\alpha$ & $m_{r}^{*}$ & $\tau$ \\
$P 4 / m n c$ & 24.4 & 2.83 & 1.71 & 0.07 \\
$P 2_{1} / n$ & 19.8 & 2.98 & 1.64 & 0.06 \\
\hline
\end{tabular}

This model is highly idealized, with the physical response of the lattice parametrized by the optical and static dielectric constants and effective-response frequency. Despite this, previous work applying this method to the hybrid perovskites has shown excellent agreement with experiment. We note that this method does not take into account other scattering processes; therefore, the results will form an upper bound for electron mobilities for a perfect crystal.

The calculated Hellwarth electron mobilities for the $P 4 / m n c$ $\left(24 \mathrm{~cm}^{2} \mathrm{~V}^{-1} \mathrm{~s}^{-1}\right)$ and $P 2_{1} / n\left(20 \mathrm{~cm}^{2} \mathrm{~V}^{-1} \mathrm{~s}^{-1}\right)$ phases of $\mathrm{Rb}_{2} \mathrm{SnI}_{6}$ are significantly smaller than those calculated for $\mathrm{Cs}_{2} \mathrm{SnI}_{6}(98$ $\mathrm{cm} \mathrm{V} \mathrm{V}^{-1} \mathrm{~s}^{-1}$ ). The increase in electron effective masses seen in the $\mathrm{Rb}_{2} \mathrm{SnI}_{6}$ phases will play a crucial role; however, when considered alone, they are insufficient to account for the dramatic difference between the experimental mobilities. Instead, the trend can be attributed to two additional factors: First, the $\mathrm{Rb}_{2} \mathrm{SnI}_{6}$ phases show considerably larger static dielectric constants $(\sim 8.4)$ than $\mathrm{Cs}_{2} \mathrm{SnI}_{6}$ (7.2), due to an increase in the ionic component of the dielectric response. Analysis of the zone center phonon eigenvalues and eigenvectors reveals that this is due to an increase in the number of low-frequency polar phonon modes that can contribute to the dielectric response of the lattice. These additional modes and their increased polarity result from the symmetry breaking in the $\mathrm{Rb}_{2} \mathrm{SnI}_{6}$ phases, which reduces the degeneracy of several modes in the highly degenerate $\mathrm{Cs}_{2} \mathrm{SnI}_{6}$ phonon spectrum. Second, the high-frequency dielectric constants of the $\mathrm{Rb}_{2} \mathrm{SnI}_{6}$ phases are slightly reduced, likely due to the changes in the iodine sublattice. Together, these factors result in significantly larger electron-phonon coupling constants $(\alpha)$ of 2.83 and 2.98 for the $P 4 / m n c$ and $P 2_{1} / n$ phases of $\mathrm{Rb}_{2} \mathrm{SnI}_{6}$, in comparison to just 1.45 for $\mathrm{Cs}_{2} \mathrm{SnI}_{6}$. The 
increased coupling, combined with larger effective masses, results in significant effective mass renormalization and small polaron relaxation times. We note that the effective-response frequency is larger in $\mathrm{Rb}_{2} \mathrm{SnI}_{6}$ than in $\mathrm{Cs}_{2} \mathrm{SnI}_{6}$, suggesting a stiffening of the lattice. Further investigation into the IR response of the modes indicates that the symmetry breaking also allows for increased polarity of the higher-frequency phonon modes, thereby increasing the effective-response frequency, with the stiffness of the lattice remaining largely unchanged.

Further examination of the phonon band structure of tetragonal $\mathrm{Rb}_{2} \mathrm{SnI}_{6}$ reveals that the tetragonal structure is dynamically stable within the harmonic approximation of forces calculated by DFPT, as evidenced by the lack of imaginary phonon modes (Figure 10). The lowest-energy optical phonon
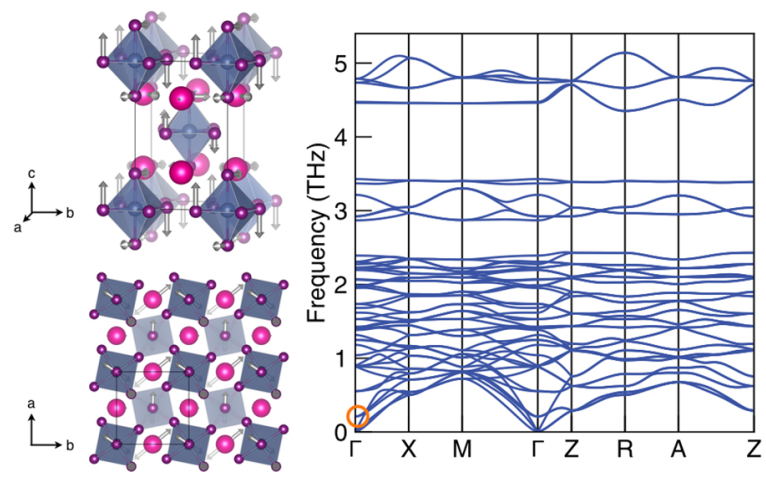

Figure 10. Phonon band structure of rubidium tin(IV) iodide in the tetragonal $P 4 / m n c$ structure $\left(a^{0} a^{0} c^{+}\right)$. The lowest-frequency optical mode at $0.25 \mathrm{THz}\left(1.03 \mathrm{meV}, 8.27 \mathrm{~cm}^{-1}\right.$ ) (denoted by the orange circle) corresponds to displacements of the rubidium and iodine atoms, as shown by the displacement vectors in the structural representations. Together, these displacements map to the octahedral tilting out of the $a b$ plane coupled with $\mathrm{Rb}^{+}$displacements observed in the lower-symmetry monoclinic structure, $\left(a^{-} a^{-} c^{+}\right)$.

occurs at $0.25 \mathrm{THz}\left(1.03 \mathrm{meV}, 8.27 \mathrm{~cm}^{-1}\right)$ at the $\Gamma$ point, as denoted by the orange circle in Figure 10. Analysis of the polarization eigenvectors indicates that this mode corresponds to displacements of the iodine and rubidium ions within the structure, which are consistent with cooperative octahedral tilting out of the $a b$ plane coupled to $\mathrm{Rb}^{+}$displacements and map onto the lower-symmetry $\left(a^{-} a^{-} c^{+}\right)$monoclinic structure observed at low temperatures. This observation is consistent with the structural behavior of $\mathrm{Rb}_{2} \mathrm{SnI}_{6}$ observed experimentally. At higher temperatures, dynamic octahedral tilting averages to the tetragonal structure observed by diffraction, yet it gives rise to the lower symmetry suggested from the local coordination environment. Upon cooling, the octahedral tilts freeze in to yield the lower-symmetry monoclinic structure observed in the crystallography. This notion is supported by previous studies of the vacancy-ordered double perovskite family, which have shown that the symmetry-lowering phase transitions in these materials are driven by condensation of the octahedral rotary phonon mode. ${ }^{65,69,70}$ The lack of imaginary modes in these calculations suggests that anharmonicity may play a role in driving the structure to the monoclinic ground state.

\section{DISCUSSION}

$\mathrm{Rb}_{2} \mathrm{SnI}_{6}$ adopts the tetragonal variant of the vacancy-ordered double perovskite structure at room temperature characterized by cooperative octahedral tilting and undergoes a symmetrylowering phase transition from tetragonal $(\mathrm{P} 4 / \mathrm{mnc})$ to monoclinic $\left(P 2_{1} / n\right)$ upon cooling. Temperature-dependent phase transitions in perovskites are typically driven by a size mismatch between the A-, B-, and X-site ions, and cooperative rotation and tilting of the octahedral units occur to improve coordination to the A-site cation by the neighboring X-site anions by collapsing the A-site void. ${ }^{4,5,18,71}$ Bond valence sum (BVS) analysis of $\mathrm{Cs}_{2} \mathrm{SnI}_{6}$ reveals that the coordination provided to the $\mathrm{Cs}^{+}$ion by the $\mathrm{I}_{12}$ cage results in a Cs valence of 1.156 , indicating that the cesium is potentially overbonded by the surrounding iodine network in the cubic structure (using tabulated parameters). This analysis provides an explanation for the observation that $\mathrm{Cs}_{2} \mathrm{SnI}_{6}$ does not appear to be susceptible to temperature-dependent phase transitions or distortions of the local coordination environment. BVS analysis of the previously reported cubic structure of $\mathrm{Rb}_{2} \mathrm{SnI}_{6}$ yields an under-bonded BVS for the $\mathrm{Rb}^{+}$ions of 0.891 , indicating that the $\mathrm{Rb}-\mathrm{I}$ bond distances are too long to provide adequate coordination to the rubidium ions in that structural model. ${ }^{61}$ The bond valence of rubidium is improved in the tetragonal (0.939) and monoclinic (1.069) structural models, consistent with the observation that $\mathrm{Rb}_{2} \mathrm{SnI}_{6}$ adopts both the tetragonal and monoclinic variants of the vacancy-ordered double perovskite family, as well as with DFT-based calculations that show the dominating role of electrostatics in determining octahedral tilting patterns in perovskite halides. ${ }^{4}$ Further, this lends support to our hypothesis that dynamic octahedral tilting occurs readily at room temperature to yield the tetragonal structure by diffraction yet reduced symmetry in the local coordination environment. This notion is further supported by analysis of the phonon spectrum of $\mathrm{Rb}_{2} \mathrm{SnI}_{6}$, which indicates that the lowest-energy optical phonon corresponds to octahedral tilting and rubidium ion displacements that together map onto the lower-symmetry monoclinic structure. Parameters used in the BVS analysis are shown in Table 5.

Table 5. Bond Valence Sum Analysis for $\mathrm{Cs}-\mathrm{I}$ and $\mathrm{Rb}-\mathrm{I}$ Bonds in $\mathrm{Cs}_{2} \mathrm{SnI}_{6}$ and $\mathrm{Rb}_{2} \mathrm{SnI}_{6}$

\begin{tabular}{lccc} 
& $B^{72}$ & $R_{0}(\AA)^{72}$ & BVS \\
$\mathrm{Cs}-\mathrm{I}(F m \overline{3} m)$ & 0.609 & 2.6926 & 1.156 \\
$\mathrm{Rb}-\mathrm{I}(F m \overline{3} m)$ & 0.638 & 2.4509 & 0.891 \\
$\mathrm{Rb}-\mathrm{I}(P 4 / m n c)$ & 0.638 & 2.4509 & 0.939 \\
$\mathrm{Rb}-\mathrm{I}\left(P 2_{1} / n\right)$ & 0.638 & 2.4509 & 1.069 \\
\hline
\end{tabular}

Analysis of the band effective masses suggests that static cooperative octahedral tilting does not play a strongly significant role in dictating the charge transport behavior in $\mathrm{Rb}_{2} \mathrm{SnI}_{6}$. While octahedral tilting in $\mathrm{Rb}_{2} \mathrm{SnI}_{6}$ induces subtle changes in the close-packed iodine framework and yields slightly larger carrier effective masses than in the cubic $\mathrm{Cs}_{2} \mathrm{SnI}_{6}$ (by a factor of 1.5-1.8), these structural changes alone are not sufficient to account for the trend in carrier mobilities observed experimentally. Rather, calculation of the electron-phonon coupling characteristics of $\mathrm{Rb}_{2} \mathrm{SnI}_{6}$ indicates that the lower symmetry due to octahedral tilting yields a larger number of low-frequency polar phonons that contribute to a large static dielectric constant and subsequently stronger electron-phonon coupling that significantly reduces carrier mobilities. Therefore, 
interpreting the electronic properties from the perspective of static cooperative octahedral tilting is insufficient to fully understand the charge transport behavior in vacancy-ordered double perovskites, and a dynamic understanding of these structural deviations is necessary to account for the observed behavior.

While lattice dynamics play a critical role in dictating the electronic properties of vacancy-ordered double perovskites, the interplay between octahedral tilting and charge transport in $\mathrm{Rb}_{2} \mathrm{SnI}_{6}$ can be generalized to a set of guiding principles for predicting charge transport behavior in vacancy-ordered double perovskites based upon the geometric model of the perovskite tolerance factor. A highly simplified use of the Goldschmidt tolerance factor, $t=\left(r_{\mathrm{A}}+r_{\mathrm{X}}\right) /\left(\sqrt{2}\left(r_{\mathrm{B}}+r_{\mathrm{X}}\right)\right)$, where $\mathrm{B}=$ $\mathrm{Sn}(\mathrm{IV})$ and $\mathrm{X}=\mathrm{I}\left(r_{\mathrm{Sn}}=0.69 \AA \text { and } r_{\mathrm{I}}=2.2 \AA\right)^{73}$ capture the observed trends in carrier mobilities for these vacancy-ordered double perovskites. Of the $\mathrm{A}_{2} \mathrm{SnI}_{6}$ family $\left(\mathrm{A}=\mathrm{Rb}^{+}, \mathrm{Cs}^{+}\right.$, $\mathrm{CH}_{3} \mathrm{NH}_{3}^{+}$, and $\left.\mathrm{CH}\left(\mathrm{NH}_{2}\right)_{2}\right)^{+}$), the tolerance factor for $\mathrm{Cs}_{2} \mathrm{SnI}_{6}$ is 0.998 and $\mathrm{Cs}_{2} \mathrm{SnI}_{6}$ exhibits the largest carrier mobility of $\sim 9$ $\mathrm{cm}^{2} \mathrm{~V}^{-1} \mathrm{~s}^{-1}$ (Figure 11). Incorporation of the larger $\mathrm{CH}_{3} \mathrm{NH}_{3}^{+}$

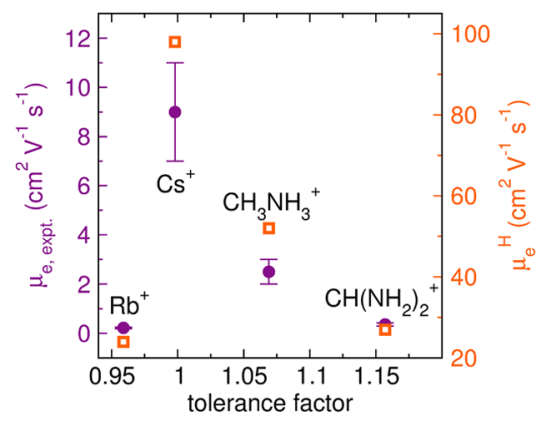

Figure 11. Experimentally $\left(\mu_{e, \text { expt. }}\right)$ and computationally derived Hellwarth $\left(\mu_{e}^{H}\right)$ electron mobilities of the $\mathrm{A}_{2} \mathrm{SnI}_{6}$ vacancy-ordered double perovskites plotted as a function of perovskite tolerance factor. Experimental electron mobilities are shown as filled purple circles on the left axis, while the calculated Hellwarth electron mobilities are denoted by open orange squares on the right axis. Values for the carrier mobilities of $\mathrm{Cs}_{2} \mathrm{SnI}_{6},\left(\mathrm{CH}_{3} \mathrm{NH}_{3}\right)_{2} \mathrm{SnI}_{6}$, and $\left(\mathrm{CH}\left(\mathrm{NH}_{2}\right)_{2}\right)_{2} \mathrm{SnI}_{6}$ are taken from a previous study. ${ }^{25}$ For $\mathrm{Rb}_{2} \mathrm{SnI}_{6}$, the $\mu_{e}^{\mathrm{H}}$ value is calculated from the tetragonal structure.

and $\mathrm{CH}\left(\mathrm{NH}_{2}\right)_{2}^{+}$ions yields tolerance factors of 1.07 and 1.16 and carrier mobilities of $\sim 2.5 \mathrm{~cm}^{2} \mathrm{~V}^{-1} \mathrm{~s}^{-1}$ and $\sim 0.36 \mathrm{~cm}^{2} \mathrm{~V}^{-1}$ $\mathrm{s}^{-1}$, respectively. ${ }^{25}$ Replacement of $\mathrm{Cs}^{+}$with $\mathrm{Rb}^{+}$yields a tolerance factor of 0.959 and a carrier mobility of $\mu_{e} \sim 0.22(3)$ $\mathrm{cm}^{2} \mathrm{~V}^{-1} \mathrm{~s}^{-1}$. Further, the trends in carrier mobilities observed experimentally are captured by the computationally derived Hellwarth electron mobilities $\left(\mu_{e}^{H}\right)$, as also shown in Figure 11 . Therefore, we might expect that vacancy-ordered double perovskite materials with tolerance factors closest to unity will exhibit weaker electron-phonon coupling interactions and higher carrier mobilities. Cations that are too large for the Asite can introduce soft, anharmonic lattice dynamics that produce tightly bound polarons that localize charge carriers and impede charge transport, as in $\left(\mathrm{CH}_{3} \mathrm{NH}_{3}\right)_{2} \mathrm{SnI}_{6}$ and $(\mathrm{CH}$ $\left.\left(\mathrm{NH}_{2}\right)_{2}\right)_{2} \mathrm{SnI}_{6}{ }^{25}$ In contrast, cations that are too small yield structures of reduced symmetry, as characterized by cooperative octahedral tilting and rotation. This slightly distorts the closepacked halogen sublattice and increases the number of lowlying phonon modes that contribute to stronger electronphonon coupling interactions and reduce carrier mobilities.

\section{CONCLUSIONS}

Replacing $\mathrm{Cs}^{+}$with the smaller $\mathrm{Rb}^{+}$ion in the vacancy-ordered double perovskite $\mathrm{Rb}_{2} \mathrm{SnI}_{6}$ is accompanied by significant changes in structural and electronic behavior. Electrical conductivity measurements of $\mathrm{Rb}_{2} \mathrm{SnI}_{6}$ indicate that, similarly to $\mathrm{Cs}_{2} \mathrm{SnI}_{6}, \mathrm{Rb}_{2} \mathrm{SnI}_{6}$ is a native $n$-type semiconductor. However, the carrier mobility is reduced by a factor of $\sim 50$ for $\mathrm{Rb}_{2} \mathrm{SnI}_{6}$ compared to $\mathrm{Cs}_{2} \mathrm{SnI}_{6}$. Structural analysis through highresolution synchrotron powder X-ray diffraction indicates that $\mathrm{Rb}_{2} \mathrm{SnI}_{6}$ crystallizes in a lower-symmetry structural modification of the vacancy-ordered double perovskite structure, characterized by cooperative tilting of the $\left[\mathrm{SnI}_{6}\right]$ octahedra $\left(a^{0} a^{0} c^{+}\right)$. Upon cooling, $\mathrm{Rb}_{2} \mathrm{SnI}_{6}$ undergoes further octahedral tilting to a monoclinic structure $\left(a^{-} a^{-} c^{+}\right)$. X-ray and neutron pair distribution function analysis reveals that the bonding environment is best described by the monoclinic structure at all temperatures, which becomes thermally averaged to higher symmetry at ambient temperature. No structural distortions are observed for $\mathrm{Cs}_{2} \mathrm{SnI}_{6}$ to $T=10 \mathrm{~K}$. Density functional calculations reveal that the reduced carrier mobilities observed experimentally in $\mathrm{Rb}_{2} \mathrm{SnI}_{6}$ relative to $\mathrm{Cs}_{2} \mathrm{SnI}_{6}$ arise from stronger electron-phonon interactions and subsequently smaller Hellwarth electron mobilities in $\mathrm{Rb}_{2} \mathrm{SnI}_{6}$ due to an increase in the number of low-frequency phonons that contribute to the dielectric response of the lattice, rather than a trivial increase in band effective mass. This observation suggests that the polaron characteristics and charge transport behavior in the vacancy-ordered double perovskite family can be tuned by introducing cooperative octahedral tilting distortions through judicious choice of A-site cation and further predicted bond valence sum and the perovskite tolerance factor.

\section{ASSOCIATED CONTENT}

\section{Supporting Information}

The Supporting Information is available free of charge on the ACS Publications website at DOI: 10.1021/acs.chemmater.8b01549.

Temperature-dependent neutron diffraction data (PDF) Crystallographic information file for tetragonal $\mathrm{Rb}_{2} \mathrm{SnI}_{6}$ at $T=295 \mathrm{~K}$ (CIF)

Crystallographic information file for monoclinic $\mathrm{Rb}_{2} \mathrm{SnI}_{6}$ at $T=100 \mathrm{~K}$ (CIF)

\section{AUTHOR INFORMATION}

\section{Corresponding Author}

*(J.R.N.) E-mail: james.neilson@colostate.edu. ORCID

Annalise E. Maughan: 0000-0002-3292-4799

David O. Scanlon: 0000-0001-9174-8601

James R. Neilson: 0000-0001-9282-5752

Notes

The authors declare no competing financial interest.

\section{ACKNOWLEDGMENTS}

This work was supported by Grant DE-SC0016083 funded by the U.S. Department of Energy, Office of Science. J.R.N. and A.E.M. acknowledge support from Research Corporation for Science Advancement through a Cottrell Scholar Award, and J.R.N. thanks the A.P. Sloan Foundation for assistance provided from a Sloan Research Fellowship. Use of the Advanced Photon 
Source at Argonne National Laboratory was supported by the U.S. Department of Energy, Office of Science, Office of Basic Energy Sciences, under Contract No. DE-AC02-06CH11357. A portion of this research at ORNL's Spallation Neutron Source was sponsored by the Scientific User Facilities Division, Office of Basic Energy Sciences, U.S. Department of Energy. This work made use of the ARCHER UK National Supercomputing Service (http://www.archer.ac.uk), via membership of the UK's HEC Materials Chemistry Consortium, which is funded by EPSRC (EP/L000202). D.O.S. acknowledges support from the EPSRC (EP/N01572X/1). D.O.S. acknowledges membership of the Materials Design Network. A.M.G. acknowledges Diamond Light Source for the cosponsorship of a studentship on the EPSRC Centre for Doctoral Training in Molecular Modelling and Materials Science (EP/L015862/1). The authors thank Dr. K. Page, Dr. D. Olds, and Dr. T.-M. Usher from the NOMAD instrument at the Spallation Neutron Source, Oak Ridge National Laboratory, and Dr. T.-M. Usher and Dr. M. McDonnell for collection of X-ray total scattering data. The authors also thank Prof. M. M. Reynolds for the use of her optical spectrometer and the expert beamline scientists at 11-BM at the Advanced Photon Source.

\section{REFERENCES}

(1) Stranks, S. D.; Snaith, H. J. Metal-halide perovskites for photovoltaic and light-emitting devices. Nat. Nanotechnol. 2015, 10, 391-402.

(2) Zhu, H.; Trinh, M. T.; Wang, J.; Fu, Y.; Joshi, P. P.; Miyata, K.; Jin, S.; Zhu, X.-Y. Organic cations might not be essential to the remarkable properties of band edge carriers in lead halide perovskites. Adv. Mater. 2017, 29, 1603072.

(3) Goldschmidt, V. M. Die gesetze der krystallochemie. Naturwissenschaften 1926, 14, 477-485.

(4) Young, J.; Rondinelli, J. M. Octahedral Rotation Preferences in Perovskite Iodides and Bromides. J. Phys. Chem. Lett. 2016, 7, 918922.

(5) Bechtel, J. S.; Van der Ven, A. Octahedral tilting instabilities in inorganic halide perovskites. Phys. Rev. Mater. 2018, 2, 025401.

(6) Walsh, A. Principles of Chemical Bonding and Band Gap Engineering in Hybrid Organic-Inorganic Halide Perovskites. J. Phys. Chem. C 2015, 119, 5755-5760.

(7) Stoumpos, C. C.; Malliakas, C. D.; Kanatzidis, M. G. Semiconducting Tin and Lead Iodide Perovskites with Organic Cations: Phase Transitions, High Mobilities, and Near-Infrared Photoluminescent Properties. Inorg. Chem. 2013, 52, 9019-9038.

(8) Amat, A.; Mosconi, E.; Ronca, E.; Quarti, C.; Umari, P.; Nazeeruddin, M. K.; Grätzel, M.; De Angelis, F. Cation-induced bandgap tuning in organohalide perovskites: Interplay of spin-orbit coupling and octahedra tilting. Nano Lett. 2014, 14, 3608-3616.

(9) Yang, Y.; Ostrowski, D. P.; France, R. M.; Zhu, K.; Van De Lagemaat, J.; Luther, J. M.; Beard, M. C. Observation of a hot-phonon bottleneck in lead-iodide perovskites. Nat. Photonics 2016, 10, 53-59.

(10) Prasanna, R.; Gold-Parker, A.; Leijtens, T.; Conings, B.; Babayigit, A.; Boyen, H.-G.; Toney, M. F.; McGehee, M. D. Band Gap Tuning via Lattice Contraction and Octahedral Tilting in Perovskite Materials for Photovoltaics. J. Am. Chem. Soc. 2017, 139, 1111711124.

(11) Linaburg, M. R.; McClure, E. T.; Majher, J. D.; Woodward, P. M. $\mathrm{Cs}_{1-x} \mathrm{Rb}_{x} \mathrm{PbCl}_{3}$ and $\mathrm{Cs}_{1-x} \mathrm{Rb}_{x} \mathrm{PbBr}_{3}$ solid solutions: Understanding octahedral tilting in lead halide perovskites. Chem. Mater. 2017, 29, 3507-3514.

(12) Zhu, X.-Y.; Podzorov, V. Charge carriers in hybrid organicinorganic lead halide perovskites might be protected as large polarons. J. Phys. Chem. Lett. 2015, 6, 4758-4761.

(13) Zhu, H.; Miyata, K.; Fu, Y.; Wang, J.; Joshi, P. P.; Niesner, D.; Williams, K. W.; Jin, S.; Zhu, X.-Y. Screening in crystalline liquids protects energetic carriers in hybrid perovskites. Science 2016, 353, 1409-1413.

(14) Neukirch, A. J.; Nie, W.; Blancon, J.-C.; Appavoo, K.; Tsai, H.; Sfeir, M. Y.; Katan, C.; Pedesseau, L.; Even, J.; Crochet, J. J.; Gupta, G.; Mohite, A. D.; Tretiak, S. Polaron stabilization by cooperative lattice distortion and cation rotations in hybrid perovskite materials. Nano Lett. 2016, 16, 3809-3816.

(15) Sendner, M.; Nayak, P. K.; Egger, D. A.; Beck, S.; Müller, C.; Epding, B.; Kowalsky, W.; Kronik, L.; Snaith, H. J.; Pucci, A.; Lovrincic, R. Optical phonons in methylammonium lead halide perovskites and implications for charge transport. Mater. Horiz. 2016, 3, 613-620.

(16) Yaffe, O.; Guo, Y.; Tan, L. Z.; Egger, D. A.; Hull, T.; Stoumpos, C. C.; Zheng, F.; Heinz, T. F.; Kronik, L.; Kanatzidis, M. G.; Owen, J. S.; Rappe, A. M.; Pimenta, M. A.; Brus, L. E. Local polar fluctuations in lead halide perovskite crystals. Phys. Rev. Lett. 2017, 118, 136001.

(17) Miyata, K.; Meggiolaro, D.; Trinh, M. T.; Joshi, P. P.; Mosconi, E.; Jones, S. C.; De Angelis, F.; Zhu, X.-Y. Large polarons in lead halide perovskites. Sci. Adv. 2017, 3, e1701217.

(18) Woodward, P. M. Octahedral tilting in perovskites. I. Geometrical considerations. Acta Crystallogr., Sect. B: Struct. Sci. $1997,53,32-43$

(19) Howard, C. J.; Stokes, H. Group-theoretical analysis of octahedral tilting in perovskites. Acta Crystallogr., Sect. B: Struct. Sci. 1998, 54, 782-789.

(20) Brown, I. D. The crystal structure of $\mathrm{K}_{2} \mathrm{TeBr}_{6}$. Can. J. Chem. 1964, 42, 2758-2767.

(21) Cai, Y.; Xie, W.; Ding, H.; Chen, Y.; Thirumal, K.; Wong, L. H.; Mathews, N.; Mhaisalkar, S. G.; Sherburne, M.; Asta, M. Computational Study of Halide Perovskite-Derived $\mathrm{A}_{2} \mathrm{BX}_{6}$ Inorganic Compounds: Chemical Trends in Electronic Structure and Structural Stability. Chem. Mater. 2017, 29, 7740-7749.

(22) Xiao, Z.; Zhou, Y.; Hosono, H.; Kamiya, T. Intrinsic Defects in a Photovoltaic Perovskite Variant $\mathrm{Cs}_{2} \mathrm{SnI}_{6}$. Phys. Chem. Chem. Phys. 2015, 17, 18900-18903.

(23) Maughan, A. E.; Ganose, A. M.; Bordelon, M. M.; Miller, E. M.; Scanlon, D. O.; Neilson, J. R. Defect tolerance to intolerance in the vacancy-ordered double perovskite semiconductors $\mathrm{Cs}_{2} \mathrm{SnI}_{6}$ and $\mathrm{Cs}_{2} \mathrm{TeI}_{6}$. J. Am. Chem. Soc. 2016, 138, 8453-8464.

(24) Saparov, B.; Sun, J.-P.; Meng, W.; Xiao, Z.; Duan, H.-S.; Gunawan, O.; Shin, D.; Hill, I. G.; Yan, Y.; Mitzi, D. B. Thin-film deposition and characterization of a Sn-deficient perovskite derivative $\mathrm{Cs}_{2} \mathrm{SnI}_{6}$. Chem. Mater. 2016, 28, 2315-2322.

(25) Maughan, A. E.; Ganose, A. M.; Candia, A. M.; Granger, J. T.; Scanlon, D. O.; Neilson, J. R. Anharmonicity and Octahedral Tilting in Hybrid Vacancy-Ordered Double Perovskites. Chem. Mater. 2018, 30, $472-483$.

(26) Lee, B.; Stoumpos, C. C.; Zhou, N.; Hao, F.; Malliakas, C.; Yeh, C.-Y.; Marks, T. J.; Kanatzidis, M. G.; Chang, R. P. Air-stable molecular semiconducting iodosalts for solar cell applications: $\mathrm{Cs}_{2} \mathrm{SnI}_{6}$ as a hole conductor. J. Am. Chem. Soc. 2014, 136, 15379-15385.

(27) Wang, J.; Toby, B. H.; Lee, P. L.; Ribaud, L.; Antao, S. M.; Kurtz, C.; Ramanathan, M.; Von Dreele, R. B.; Beno, M. A. A dedicated powder diffraction beamline at the Advanced Photon Source: Commissioning and early operational results. Rev. Sci. Instrum. 2008, 79, 085105.

(28) Larson, A. C.; Von Dreele, R. B. GSAS, General Structure Analysis System; Los Alamos National Laboratory: Los Alamos, NM, 1994.

(29) Toby, B. H. EXPGUI, a graphical user interface for GSAS. J. Appl. Crystallogr. 2001, 34, 210-213.

(30) Momma, K.; Izumi, F. VESTA3 for three-dimensional visualization of crystal, volumetric and morphology data. J. Appl. Crystallogr. 2011, 44, 1272-1276.

(31) Chupas, P. J.; Qiu, X.; Hanson, J. C.; Lee, P. L.; Grey, C. P.; Billinge, S. J. Rapid-acquisition pair distribution function (RA-PDF) analysis. J. Appl. Crystallogr. 2003, 36, 1342-1347.

(32) Hammersley, A.; Svensson, S.; Hanfland, M.; Fitch, A.; Hausermann, D. Two-dimensional detector software: From real 
detector to idealised image or two-theta scan. High Pressure Res. 1996, $14,235-248$.

(33) Qiu, X.; Thompson, J. W.; Billinge, S. J. PDFgetX2: a GUIdriven program to obtain the pair distribution function from $\mathrm{X}$-ray powder diffraction data. J. Appl. Crystallogr. 2004, 37, 678-678.

(34) Farrow, C.; Juhas, P.; Liu, J.; Bryndin, D.; Božin, E.; Bloch, J.; Proffen, T.; Billinge, S. PDFfit2 and PDFgui: computer programs for studying nanostructure in crystals. J. Phys.: Condens. Matter 2007, 19, 335219 .

(35) Neuefeind, J.; Feygenson, M.; Carruth, J.; Hoffmann, R.; Chipley, K. K. The nanoscale ordered materials diffractometer NOMAD at the spallation neutron source SNS. Nucl. Instrum. Methods Phys. Res., Sect. B 2012, 287, 68-75.

(36) Kresse, G.; Hafner, J. Ab Initio Molecular Dynamics for Liquid Metals. Phys. Rev. B: Condens. Matter Mater. Phys. 1993, 47, 558-561.

(37) Kresse, G.; Hafner, J. Ab Initio Molecular-Dynamics Simulation of the Liquid-Metal Amorphous-Semiconductor Transition in Germanium. Phys. Rev. B: Condens. Matter Mater. Phys. 1994, 49, 14251-14269.

(38) Kresse, G.; Furthmüller, J. Efficiency of $A b$ Initio Total Energy Calculations for Metals and Semiconductors Using a Plane Wave Basis Set. Comput. Mater. Sci. 1996, 6, 15-50.

(39) Kresse, G.; Furthmüller, J. Efficient Iterative Schemes for $A b$ Initio Total-Energy Calculations Using a Plane-Wave Basis Set. Phys. Rev. B: Condens. Matter Mater. Phys. 1996, 54, 11169-11186.

(40) Kresse, G.; Joubert, D. From Ultrasoft Pseudopotentials to the Projector Augmented-Wave Method. Phys. Rev. B: Condens. Matter Mater. Phys. 1999, 59, 1758-1775.

(41) Perdew, J. P.; Ruzsinszky, A.; Csonka, G. I.; Vydrov, O. A.; Scuseria, G. E.; Constantin, L. A.; Zhou, X.; Burke, K. Restoring the Density-Gradient Expansion for Exchange in Solids and Surfaces. Phys. Rev. Lett. 2008, 100, 136406.

(42) Perdew, J. P.; Burke, K.; Ernzerhof, M. Generalized Gradient Approximation Made Simple. Phys. Rev. Lett. 1996, 77, 3865-3868.

(43) Krukau, A. V.; Vydrov, O. A.; Izmaylov, A. F.; Scuseria, G. E. Influence of the Exchange Screening Parameter on the Performance of Screened Hybrid Functionals. J. Chem. Phys. 2006, 125, 224106.

(44) Ganose, A. M.; Savory, C. N.; Scanlon, D. O. Electronic and defect properties of $\left(\mathrm{CH}_{3} \mathrm{NH}_{3}\right)_{2} \mathrm{~Pb}(\mathrm{SCN})_{2} \mathrm{I}_{2}$ analogues for photovoltaic applications. J. Mater. Chem. A 2017, 5, 7845-7853.

(45) Biswas, D.; Ganose, A. M.; Yano, R.; Riley, J.; Bawden, L.; Clark, O.; Feng, J.; Collins-Mcintyre, L.; Sajjad, M.; Meevasana, W.; Hoesch, M.; Rault, J.; Sasagawa, T.; Scanlon, D.; King, P.; Kim, T. K. Narrowband anisotropic electronic structure of $\operatorname{ReS}_{2}$. Phys. Rev. B: Condens. Matter Mater. Phys. 2017, 96, 085205.

(46) Bradley, C.; Cracknell, A. The mathematical theory of symmetry in solids: representation theory for point groups and space groups; Oxford University Press: 2010.

(47) Heyd, J.; Scuseria, G. E.; Ernzerhof, M. Hybrid functionals based on a screened Coulomb potential. J. Chem. Phys. 2003, 118, 82078215.

(48) Heyd, J.; Scuseria, G. E.; Ernzerhof, M. Erratum: "Hybrid functionals based on a screened Coulomb potential"[J. Chem. Phys. 118, 8207 (2003)]. J. Chem. Phys. 2006, 124, 219906.

(49) Hobbs, D.; Kresse, G.; Hafner, J. Fully unconstrained noncollinear magnetism within the projector augmented-wave method. Phys. Rev. B: Condens. Matter Mater. Phys. 2000, 62, 11556-11570.

(50) Ganose, A. M.; Butler, K. T.; Walsh, A.; Scanlon, D. O. Relativistic electronic structure and band alignment of BiSI and BiSeI: candidate photovoltaic materials. J. Mater. Chem. A 2016, 4, 20602068.

(51) Savory, C. N.; Ganose, A. M.; Scanlon, D. O. Exploring the PbS$\mathrm{Bi}_{2} \mathrm{~S}_{3}$ series for next generation energy conversion materials. Chem. Mater. 2017, 29, 5156-5167.

(52) https://github.com/SMTG-UCL/CSI-CTI (accessed Feb. 1, 2016).
(53) Baroni, S.; De Gironcoli, S.; Dal Corso, A.; Giannozzi, P. Phonons and related crystal properties from density-functional perturbation theory. Rev. Mod. Phys. 2001, 73, 515.

(54) Gajdoš, M.; Hummer, K.; Kresse, G.; Furthmüller, J.; Bechstedt, F. Linear optical properties in the projector-augmented wave methodology. Phys. Rev. B: Condens. Matter Mater. Phys. 2006, 73, 045112 .

(55) https://github.com/WMD-group/MacroDensity (accessed Mar. 13, 2018)

(56) https://github.com/jarvist/PolaronMobilityFeynmanKadanoffOsakaHellwarth (accessed July 26, 2017).

(57) Frost, J. M. Calculating polaron mobility in halide perovskites. Phys. Rev. B: Condens. Matter Mater. Phys. 2017, 96, 195202.

(58) Hellwarth, R. W.; Biaggio, I. Mobility of an electron in a multimode polar lattice. Phys. Rev. B: Condens. Matter Mater. Phys. 1999, 60, 299-307.

(59) Kadanoff, L. P. Boltzmann Equation for Polarons. Phys. Rev. 1963, 130, 1364-1369.

(60) Feynman, R. P.; Hellwarth, R. W.; Iddings, C. K.; Platzman, P. M. Mobility of Slow Electrons in a Polar Crystal. Phys. Rev. 1962, 127, 1004-1017.

(61) Werker, W. Die Krystallstruktur des $\mathrm{Rb}_{2} \mathrm{SnI}_{6}$ und $\mathrm{Cs}_{2} \mathrm{SnI}_{6}$. Recl. Trav. Chim. Pays-Bas 1939, 58, 257-258.

(62) Boysen, H.; Hewat, A. W. A neutron powder investigation of the structural changes in $\mathrm{K}_{2} \mathrm{SnCl}_{6}$. Acta Crystallogr., Sect. B: Struct. Crystallogr. Cryst. Chem. 1978, 34, 1412-1418.

(63) Abrahams, S.; Ihringer, J.; Marsh, P. Structural and thermal dependence of normal-mode condensations in $\mathrm{K}_{2} \mathrm{TeBr}_{6}$. Acta Crystallogr., Sect. B: Struct. Sci. 1989, 45, 26-34.

(64) McQueeney, R. Dynamic radial distribution function from inelastic neutron scattering. Phys. Rev. B: Condens. Matter Mater. Phys. 1998, 57, 10560.

(65) Cooke, D. F.; Armstrong, R. L. Investigation of the Rotary Lattice Mode in $\mathrm{R}_{2} \mathrm{PtCl}_{6}$ Compounds. I. From Measurements of the ${ }^{35} \mathrm{Cl}$ Nuclear Quadrupole Resonance Frequency. Can. J. Phys. 1971, 49, 2381-2388.

(66) Beecher, A. N.; Semonin, O. E.; Skelton, J. M.; Frost, J. M.; Terban, M. W.; Zhai, H.; Alatas, A.; Owen, J. S.; Walsh, A.; Billinge, S. J. Direct observation of dynamic symmetry breaking above room temperature in methylammonium lead iodide perovskite. ACS Energy Lett. 2016, 1, 880-887.

(67) Yang, R. X.; Skelton, J. M.; da Silva, L.; Frost, J. M.; Walsh, A. Spontaneous Octahedral Tilting in the Cubic Inorganic Caesium Halide Perovskites $\mathrm{CsSnX}_{3}$ and $\mathrm{CsPbX}_{3}(\mathrm{X}=\mathrm{F}, \mathrm{Cl}, \mathrm{Br}, \mathrm{I})$. J. Phys. Chem. Lett. 2017, 8, 4720-4726.

(68) Laurita, G.; Fabini, D. H.; Stoumpos, C. C.; Kanatzidis, M. G.; Seshadri, R. Chemical tuning of dynamic cation off-centering in the cubic phases of hybrid tin and lead halide perovskites. Chem. Sci. 2017, $8,5628-5635$.

(69) O'Leary, G.; Wheeler, R. Phase transitions and soft librational modes in cubic crystals. Phys. Rev. B 1970, 1, 4409.

(70) Waschk, V.; Pelzl, J.; Höck, K.-H.; Eckold, G. Inelastic neutron scattering from transverse acoustic modes in $\mathrm{K}_{2} \mathrm{SnCl}_{6}$. Solid State Commun. 1984, 52, 829-831.

(71) Woodward, P. M. Octahedral tilting in perovskites. II. Structure stabilizing forces. Acta Crystallogr., Sect. B: Struct. Sci. 1997, 53, 44-66.

(72) Brown, I.; Altermatt, D. Bond-valence parameters obtained from a systematic analysis of the inorganic crystal structure database. Acta Crystallogr., Sect. B: Struct. Sci. 1985, 41, 244-247.

(73) Shannon, R. D. Revised effective ionic radii and systematic studies of interatomic distances in halides and chalcogenides. Acta Crystallogr., Sect. A: Cryst. Phys., Diffr., Theor. Gen. Crystallogr. 1976, 32, $751-767$. 